\title{
A thermodynamically consistent cohesive-frictional interface model for mixed mode delamination
}

\author{
Francesco Parrinello ${ }^{\mathrm{a}, *}$, Giuseppe Marannano ${ }^{\mathrm{b}}$, Guido Borino ${ }^{\mathrm{a}}$, \\ ${ }^{a}$ Dipartimento di Ingegneria Civile, Ambientale, Aerospaziale, dei Materiali. \\ Università di Palermo, Viale delle Scienze, Ed. 8, 90128 Palermo \\ ${ }^{b}$ Dipartimento di Ingegneria Chimica, Gestionale, Informatica, Meccanica. \\ Università di Palermo, Viale delle Scienze, Ed. 8, 90128 Palermo
}

\begin{abstract}
A new interface constitutive model based on damage mechanics and frictional plasticity theory is presented. The model is thermodynamically consistent, it is able to accurately reproduce arbitrary mixed mode debonding conditions and it is proved that the separation work is always bounded between the fracture energy in mode I and the fracture energy in mode II. Analytical results are given for proportional loading paths and for two non-proportional loading paths, confirming the correct behaviour of the model for complex loading histories. Numerical and analytical solutions are compared for three classical delamination tests and frictional effects on $4 \mathrm{ENF}$ are also considered.
\end{abstract}

Keywords:

cohesive-frictional interface, mixed-mode delamination, thermodynamics.

\section{1. Introduction}

2 The challenge of modeling the progressive formation, development and prop-

3 agation of displacement discontinuities, such as fracture or delamination phe-

4 nomena, has been successfully faced by the introduction of Cohesive Zone Mod-

5 els (CZMs). CZMs are constitutive non-linear relations able to model the tran-

\footnotetext{
* corresponding author

Email addresses: francesco.parrinello@unipa.it (Francesco Parrinello), giuseppe marannano@unipa.it (Giuseppe Marannano), guido.borino@unipa.it ( Guido Borino)
} 
- sition between virgin material, partially broken ligaments and full opening along

7 a surface. The first pioneering papers goes back to the early 60s by Dugdale

\& (1960) and Barenblatt (1962) who established the physical basis of the constitu-

9 tive framework. Since then many developments have been done, with the main

10 assessment of joining the surface cohesive constitutive model with the concept

11 of interface elements (Xu and Needleman, 1993; Allix et al., 1995; Alfano and

12 Crisfield, 2001). The introduction of non-linear interface elements in conjunc-

13 tion with standard Finite Element analysis gave rise to one of the most powerful

14 approach to non-linear structural failure analysis.

15 In the last years, several crucial aspects have been accurately investigated

16 such as: isotropic and orthotropic cohesive interface models (Corigliano and

17 Allix, 2000), coupling damage and plasticity (Spada et al., 2009; Kolluri et al.,

18 2014), different mode I and mode II fracture energies (Alfano and Crisfield,

19 2001; Benzerga et al., 2008), thermodynamic consistency (Parrinello et al., 2009;

20 Mosler and Scheider, 2011; Guiamatsia and Nguyen, 2014; Serpieri et al., 2015),

21 time dependent and viscous effects (Corigliano and Ricci, 2001; Giambanco and

22 Fileccia Scimemi, 2006; Zreid et al., 2013; Musto and Alfano, 2013)

23 Recently, special attention has been focused on the assessment of an energy

24 rational behavior of interfaces models loaded under arbitrary mixed mode con-

25 ditions. It is actually required a physical sound behavior for monotonic and

26 cyclic loading for different mixity rate loadings. It is also of paramount interest

${ }_{27}$ to ensure, for any loading path, the satisfaction of thermodynamic principles.

$28 \quad$ In order to control mixed loading in traction and shearing Park et al. (2009)

29 proposed a potential-based cohesive zone model for mixed-mode fracture, which

so is defined as a particle debonding potential at the material point level. The

31 model is based on a unique potential which is function of both normal and

32 tangential component of the separation displacement. The work of separa-

33 tion is evaluated for some loading paths, producing physically consistent re-

34 sults. On the contrary, Park et al. (2009) show that, when the mode I and

35 mode II fracture energies are $G_{I I}>G_{I}$, the potential based of Xu and Needle-

36 man (1993) produces work of separation, $W_{T}$, in a mixed mode loading paths 
which is $W_{T}>G_{I I}>G_{I}$, in disagreement with experimental evidence (see Benzeggagh and Kenane (1996)). Analogously, when $G_{I I}<G_{I}$, the separation work in a mixed mode loading path is $W_{T}<G_{I I}<G_{I}$.

41 mixed mode separation loading paths and under over-closure conditions showing 42 some shortcoming of the model of Xu and Needleman (1993).

43 Dimitri et al. (2014) carefully evaluate the response performances of four 44 well-known interface constitutive models under mixed mode loading and whether 45 they are always consistent in terms of stress and energy dissipation. In Dim46 itri et al. (2014), the authors show that the CZMs proposed by McGarry et al. 47 (2014); Högberg (2006); Camanho et al. (2003), under particular mixed loading 48 condition, may produce unphysical results. Moreover, in the model of van den 49 Bosch et al. (2006) the unloading law, different than the loading one, is not so explicitly defined and energy dissipation can not be directly evaluated. In such 51 model two independent laws are defined respectively for the tangential traction 52 component and for the normal one and, as already stated in Mosler and Scheider 53 (2011), symmetry the tangent stiffness matrix is not achieved.

${ }_{54}$ On the basis of the above criticism, Dimitri et al. (2014) propose a ther55 modynamically consistent model, defined as an improvement of the van den 56 Bosch et al. (2006) model, but derived by an Helmholtz free energy function. 57 The same tangential and normal traction interface laws of van den Bosch et al. 58 (2006) are rigorously derived by applying the Coleman and Noll (1963) proce59 dure. The cost of such achievement is the necessity of employ four independent 6o scalar damage variables. Mosler and Scheider (2011) pointed out the relevance 61 of the thermodynamical consistency for finite strains and anisotropic interface 62 models.

${ }_{63}$ In Serpieri et al. (2015) a thermodynamic consistent cohesive frictional model 64 with different mode I and mode II fracture energies is presented. The model is 65 defined by means of a single scalar damage variable and a single scalar equivalent 66 displacement. The authors prove, that under the above hypothesis the total ${ }_{67}$ dissipation of energy, which is equal to the separation work, in pure mode I $\left(G_{I}\right)$ 
75 (2006); Parrinello et al. (2009); Spada et al. (2009); Sacco and Lebon (2012);

76 Guiamatsia and Nguyen (2014)). The contribution of frictional behaviour to the

77 the mode II dissipation energy has been analyzed under increasing cycling load

78 in Parrinello et al. (2013) by the cohesive-frictional interface model proposed in

79 Parrinello et al. (2009).

81 namically consistent, with two different fracture energies in mode I and in mode

82 II, which behaves also correctly under any cyclic loading in mixed mode, is, in 83 the authors' knowledge, a goal not yet reached.

84 In the present paper a new thermodynamically consistent CZM is proposed.

${ }_{85}$ It is based on a predefined Helmholtz free energy density with a single scalar

86 damage variable and it produces two independent work of separation in pure

87 mode I and pure mode II delamination conditions. The proposed model can also

88 take in to account frictional effects with a smooth transition of the mechanical

s9 behavior, from the initial elastic one of the virgin material, to the fully debonded

so behavior with frictional residual strength. The cohesive-frictional behavior is

91 based on the same mesoscale interpretation of the scalar damage variable, pre-

92 viously proposed in Alfano and Sacco (2006); Parrinello et al. (2009). In fact,

93 the model proposed in this paper can be considered as a rational evolution of

94 the interface model developed by Parrinello et al. (2009), whose main limit is

95 that it produces a unique separation work, excluding the presence of frictional

96 effects, independently of the debonding mode condition. The proposed formu-

97 lation is defined by a new damage activation function. Traction components,

98 damage evolution and the relevant constitutive equations are derived by follow- 
ing classical thermodynamic arguments (Coleman and Noll, 1963). The model implicitly verify the second thermodynamic law by proving that dissipation is non-negative for every loading path; it produces two independent fracture energies in pure mode I and pure mode II debonding conditions and produces physically consistent results under mixed mode debonding ones.

The paper is organized as follows: the new model is presented in Section 2. The solutions of monotonic and non-proportional loading paths are analytically derived in respectively in Section 3 and in Section 4. Numerical results of three delamination tests are compared with the relevant analytical solutions in Section 5 and, finally, closing remarks are reported in section 6 .

\section{The cohesive-frictional model with different fracture energy in} mode $I$ and in mode II

Damage mechanics concepts are widely used for cohesive interface models (Corigliano, 1993; Daudeville et al., 1995; Allix et al., 1995; Corigliano and Allix, 2000; Mosler and Scheider, 2011) since they possess all the necessary features to properly describe cohesive fracture processes.

As recently pointed out for damage based interface constitutive models (Alfano and Sacco, 2006; Parrinello et al., 2009; Spada et al., 2009; Serpieri and

Alfano, 2011; Guiamatsia and Nguyen, 2014) effective formulations can be derived considering the classical scalar damage variable $\omega$ in a geometrical setting as

$$
\omega:=\frac{\mathrm{d} S_{c}}{\mathrm{~d} S}=\frac{\mathrm{d} S-\mathrm{d} S_{s}}{\mathrm{~d} S}
$$

where, at a generic point, the reference interface surface $\mathrm{d} S$ is associated to a sound (virgin) fraction, $\mathrm{d} S_{s}$ and to a complementary cracked fraction $\mathrm{d} S_{c}$ (see Figure 1 a))

Adopting a mixture approach, at the sub-scale where the two fractions are defined, a specific kinematic, static and constitutive relations can be established, which are then reported at the macro interface level. Since interface are used to 


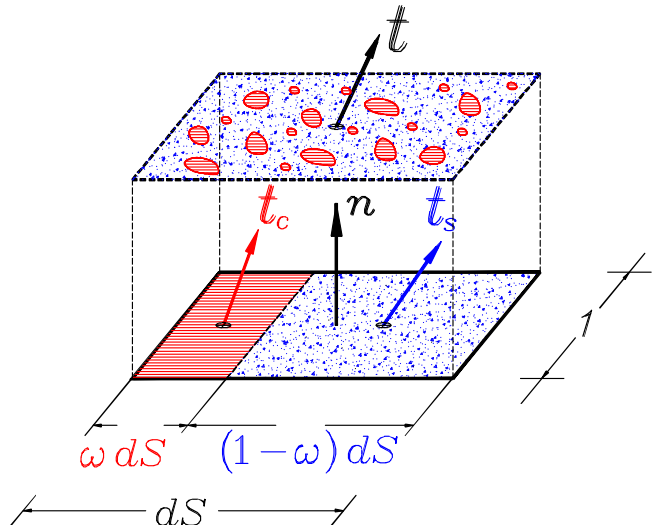

a)

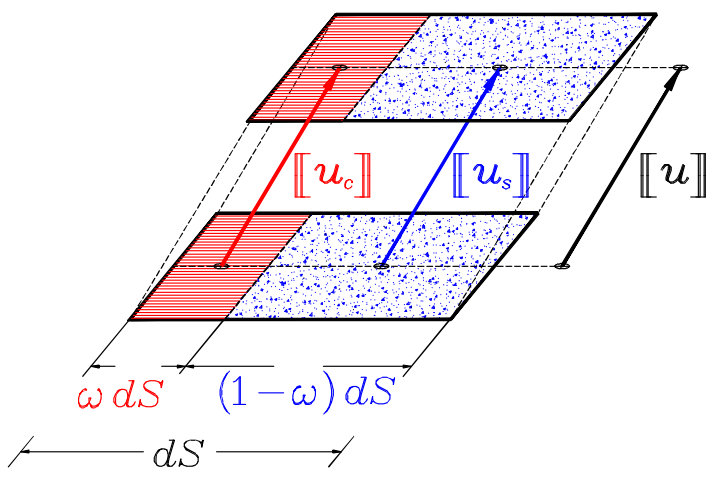

b)

Figure 1: Geometrical sketch showing the decomposition in two fractions: a) Decomposition of the damaged surface $\mathrm{d} S$ in the cracked fraction $\omega \mathrm{d} S$ and the sound fraction $(1-\omega) \mathrm{d} S$ with the respective traction vectors $\boldsymbol{t}_{c}$ and $\boldsymbol{t}_{s}$. By the equilibrium condition $\boldsymbol{t}_{s}+\boldsymbol{t}_{c}=\boldsymbol{t}$. b) kinematic representation of the displacement jump $\llbracket \boldsymbol{u} \rrbracket$, with $\llbracket \boldsymbol{u}_{c} \rrbracket$ and $\llbracket \boldsymbol{u}_{s} \rrbracket$ jump displacement vectors across the cracked and the sound fractions. By kinematic consistency $\llbracket \boldsymbol{u} \rrbracket=\llbracket \boldsymbol{u}_{c} \rrbracket=\llbracket \boldsymbol{u}_{s} \rrbracket$. 


$$
\boldsymbol{u}_{c}=\boldsymbol{\delta}_{c}^{e}+\boldsymbol{\delta}_{c}^{p}+\boldsymbol{\delta}_{c}^{d}
$$

145 where $\boldsymbol{\delta}_{c}^{e}$ is the elastic component (due to micro elastic deformation modes in 146 contact and/or in sliding), $\delta_{c}^{p}$ is the plastic component (due to to frictional ${ }_{147}$ deformation modes including dilatancy), and $\boldsymbol{\delta}_{c}^{d}$ is the detachment component 148 or gap vector (due to opening or even sliding without compressive state).

The displacement jump contributions defined in Eq. (4) have also to satisfy some kinematic conditions related to the unilateral contact (for opening/closing 
conditions), as well as for frictional effects. All the components jump vectors have a Cartesian component along the normal to the interface oriented from the $(-)$ lower surface to the $(+)$ upper surface, which is denoted by an extra index $n$, and a tangential component along the interface denoted by an extra index $t$.

Considering the cracked fraction, the normal elastic component $\delta_{c n}^{e}$ has to be non-positive, since it is active only in a contact compressive state. Conversely, the detachment normal component, $\delta_{c n}^{d}$, has to be non-negative, since it describe the opening mechanism. Moreover, the two quantities $\delta_{c n}^{e}$ and $\delta_{c n}^{d}$ cannot be both different from zero at the same time. As a conclusion the following classic elastic contact complementarity conditions holds

$$
\delta_{c n}^{e} \leq 0, \quad \delta_{c n}^{d} \geq 0, \quad \delta_{c n}^{e} \delta_{c n}^{d}=0
$$

$$
\text { Considering the elastic tangential component, } \delta_{c t}^{e} \text {, it is observed that no sign }
$$
restriction is required, but it has also to satisfy the mutual activation condition in the form

$$
\delta_{c t}^{e} \delta_{c n}^{d}=0
$$

Finally, no sign restriction are imposed on the detachment tangential component $\boldsymbol{\delta}_{c t}^{d}$, which means that in case of re-closing deformation mode tangential components previously produced in a opening state, may be accounted for, $\left(\delta_{c t}^{d} \neq 0\right)$.

As a final remark, it can be easily proved that Eqs. (2)-(6) hold also if written in rate form.

\subsection{Thermodynamic consistency}

In order to comply thermodynamic principles, the Helmholtz free energy density function (for unit surface) is introduced, playing the role of potential with respect to the state variables, either external, or internal ones. Since the adopted model is based on the superposition of two fractions, in which the sound fraction is weighted by the integrity coefficient $(1-\omega)$, whereas the cracked fraction is weighted by the damage coefficient $\omega$, it follows that the Helmholtz free energy can be given in the following form: 


$$
\psi\left(\boldsymbol{\delta}_{s}^{e}, \boldsymbol{\delta}_{c}^{e}, \omega, \xi\right)=(1-\omega) \bar{\psi}_{s}^{e}\left(\boldsymbol{\delta}_{s}^{e}\right)+\omega \bar{\psi}_{c}^{e}\left(\boldsymbol{\delta}_{c}^{e}\right)+\psi_{i}(\xi)
$$

178

where $\bar{\psi}_{s}^{e}$ and $\bar{\psi}_{c}^{e}$ are the elastic free energy densities for the unweighted sound and cracked fractions, both function of the respective elastic deformations. $\psi_{i}$ is the internal free energy related to a scalar internal variable $\xi$, introduced for a specific description of the post-peak traction - relative displacement regime (softening).

In what follow linear elasticity behavior is assumed, either for the sound, or for the cracked fraction, which implies a quadratic form for the two elastic free energies, namely

$$
\bar{\psi}_{s}^{e}\left(\boldsymbol{\delta}_{s}^{e}\right)=\frac{1}{2} \boldsymbol{\delta}_{s}^{e T} \boldsymbol{K}_{s} \boldsymbol{\delta}_{s}^{e} ; \quad \bar{\psi}_{c}^{e}\left(\boldsymbol{\delta}_{c}^{e}\right)=\frac{1}{2} \boldsymbol{\delta}_{c}^{e T} \boldsymbol{K}_{c} \boldsymbol{\delta}_{c}^{e} ;
$$

Equations (8) give the stored strain energies of the two fractions each of which, in agreement with Eq. (7), is weighted by the coefficients $(1-\omega)$ and $\omega$ respectively.

$\boldsymbol{K}_{s}$ and $\boldsymbol{K}_{c}$ are two positive definite diagonal stiffness matrices in which $K_{i}^{s}$ and $K_{i}^{c}$ are positive stiffness coefficients and the index $(i=n, t)$ stands for normal and tangential component.

Thermodynamic consistency, in the form of the second principle, can be enforced by the Clausius-Duhem inequality, which gives an explicit form for the non-negative mechanical energy dissipation density:

$$
D=\boldsymbol{t}^{T} \dot{\boldsymbol{u}}-\dot{\psi} \geq 0
$$

Expanding $\dot{\psi}$, considering the specific form given in Eqs. (7) and (8), and making also use of the decomposition of the total interface strains, given in Eqs. (3) and (4) written in the following rate form: $\dot{\boldsymbol{\delta}}_{s}^{e}=\dot{\boldsymbol{u}}$ and $\dot{\boldsymbol{\delta}}_{c}^{e}=\dot{\boldsymbol{u}}-\dot{\boldsymbol{\delta}}_{c}^{p}-\dot{\boldsymbol{\delta}}_{c}^{d}$, gives

$$
D=\left(\boldsymbol{t}-\frac{\partial \psi}{\partial \boldsymbol{\delta}_{s}^{e}}-\frac{\partial \psi}{\partial \boldsymbol{\delta}_{c}^{e}}\right)^{T} \dot{\boldsymbol{u}}+\left(\frac{\partial \psi}{\partial \boldsymbol{\delta}_{c}^{e}}\right)^{T}\left(\dot{\boldsymbol{\delta}}_{c}^{p}+\dot{\boldsymbol{\delta}}_{c}^{d}\right)-\frac{\partial \psi}{\partial \omega} \dot{\omega}-\frac{\partial \psi_{i n}}{\partial \xi} \dot{\xi} \geq 0
$$

No dissipation $(D=0)$ is produced in the case of any purely reversible deformation modes (elasticity) in which $\dot{\omega}=\dot{\xi}=0$ and $\dot{\boldsymbol{\delta}}_{c}^{p}=\dot{\boldsymbol{\delta}}_{c}^{d}=\mathbf{0}$, which gives as 
result

$$
\boldsymbol{t}=\underbrace{\frac{\partial \psi}{\partial \boldsymbol{\delta}_{s}^{e}}}_{\boldsymbol{t}_{s}}+\underbrace{\frac{\partial \psi}{\partial \boldsymbol{\delta}_{c}^{e}}}_{\boldsymbol{t}_{c}}
$$

201

211 and

here it has been set

$$
\boldsymbol{t}_{s}:=\frac{\partial \psi}{\partial \boldsymbol{\delta}_{s}^{e}}=(1-\omega) \boldsymbol{K}_{s} \boldsymbol{\delta}_{s}^{e} ; \quad \boldsymbol{t}_{c}:=\frac{\partial \psi}{\partial \boldsymbol{\delta}_{c}^{e}}=\omega \boldsymbol{K}_{c} \boldsymbol{\delta}_{c}^{e}
$$

The two tractions, $\boldsymbol{t}_{s}$ and $\boldsymbol{t}_{c}$ (See Figure 1 a) play the role of traction vectors acting on each of the two fractions of the model and the relation $\boldsymbol{t}=\boldsymbol{t}_{s}+\boldsymbol{t}_{c}$ is a form of internal linear momentum balance equation.

Following a well established procedure, the state equation (11) holds also for dissipative deformation processes, so that the dissipation function can be rewritten as

$$
D=Y \dot{\omega}+\boldsymbol{t}_{c}^{T} \dot{\boldsymbol{\delta}}_{c}^{p}-\chi \dot{\xi} \geq 0 .
$$

where the orthogonality condition $\boldsymbol{t}_{c}^{T} \dot{\boldsymbol{\delta}}_{c}^{d}=0$ has been used. The energy release rate $Y$ and the static like conjugate internal variable $\chi$ introduced in Eq. (13) complete the set of state equations defined as

$$
\begin{aligned}
Y & =-\frac{\partial \psi}{\partial \omega}=\bar{\psi}_{s}^{e}-\bar{\psi}_{c}^{e} \\
& =\frac{1}{2} \boldsymbol{\delta}_{s}^{e T} \boldsymbol{K}_{s} \boldsymbol{\delta}_{s}^{e}-\frac{1}{2} \boldsymbol{\delta}_{c}^{e T} \boldsymbol{K}_{c} \boldsymbol{\delta}_{c}^{e}
\end{aligned}
$$

12 Equation (13) states that the total dissipation $D$ is given by a first term 213 related to the energy for a possible increment of damage $Y \dot{\omega}$, a second term for ${ }_{214}$ a possible frictional mechanism $\boldsymbol{t}_{c}^{T} \dot{\boldsymbol{\delta}}_{c}^{p}$ (including dilatancy effects) and finally 215 the third (negative) term $\chi \dot{\xi}$ is the rate energy spent in the reorganizing the 216 internal microstructure for the evolution of the softening behavior. Moreover, 217 observing Eq. (14) it can be stated that the energy release rate $Y$ is given as 218 the strain energy in the sound fraction minus the strain energy of the cracked 219 fraction, the latter being not available for further damage increments. 
A further relevant feature shown by Eq.(13) is that there are no dissipative interactions between damage and friction modes. This uncoupled structure means that a damage increment (decohesion growth) does not necessarily

${ }_{223}$ requires a change in the frictional state and, of course, the vice-versa. Equa224 tion (13) can then be split as

$$
D=D_{d}+D_{p}
$$

225

tional increment, given as

$$
\begin{aligned}
D_{d}(\dot{\omega}, \dot{\xi}) & =Y \dot{\omega}-\chi \dot{\xi} \geq 0 \\
D_{p}\left(\dot{\boldsymbol{\delta}}_{c}^{p}\right) & =\boldsymbol{t}_{c}^{T} \dot{\boldsymbol{\delta}}_{c}^{p} \geq 0
\end{aligned}
$$

$$
\phi_{d}(Y, \chi ; \boldsymbol{u})=Y-\chi-\bar{Y}(\boldsymbol{u})-Y_{0} \leq 0
$$

${ }^{234}$ in which $Y_{0}$ is a positive constant value accounting for the initial unloaded dam235 age threshold; the internal variable $\chi$ describes the damage threshold increment, 236 $\dot{\chi} \geq 0$ due to the damage evolution, and finally $\bar{Y}(\boldsymbol{u})$ is a positive term given as ${ }_{237}$ function of the kinematic state

$$
\bar{Y}(\boldsymbol{u})=\frac{1}{2} \boldsymbol{u}^{T} \boldsymbol{A} \boldsymbol{u}=\frac{1}{2} A_{n} u_{n}^{2}+\frac{1}{2} A_{t} u_{t}^{2}
$$


conditions can be obtained as

$$
\begin{aligned}
\dot{\omega} & =\frac{\partial \phi_{d}}{\partial Y} \dot{\lambda}_{d}=\dot{\lambda}_{d}, \\
\dot{\xi} & =-\frac{\partial \phi_{d}}{\partial \chi} \dot{\lambda}_{d}=\dot{\lambda}_{d}, \\
\dot{\lambda}_{d} & \geq 0, \quad \phi_{d} \dot{\lambda}_{d}=0, \quad \dot{\phi}_{d} \dot{\lambda}_{d}=0
\end{aligned}
$$

${ }_{241}$ where $\dot{\lambda}_{d}$ is the damage multiplier.

242 Unlike what presented previously by the authors (Parrinello et al., 2009), ${ }_{243}$ where the damage activation function is driven only by $Y$, producing the same ${ }_{244}$ separation work in pure model $I$, in mode $I I$ and for any mixed mode, in the 245 present paper a new formulation is proposed, which is enhanced by the inser246 tion of a state displacement dependent damage activation function, as shown 247 by Eq.(18). This new approach, even if still based on a single scalar damage 248 variable, produces a different separation work in pure mode $I$ and in pure mode $I I$, and as it will be shown in the next Section does not suffer of any inconsistency in mixed modes. The values of the two new constitutive parameters, $A_{n}$ ${ }_{251}$ and $A_{t}$, are related to the values of the Fracture Energies, $G_{I}$ and $G_{I I}$. If it 252 is assumed $G_{I I}>G_{I}$, as it is usually shown by experimental evidences, it is necessary to set $A_{t}>A_{n}$. Otherwise, in the case of $G_{I}>G_{I I}$ the parameters have to be set as $A_{n}>A_{t}$. Details on choice and on the physical meaning of the two constants will be given in the next Section.

The dissipation associated with the damage activation can be computed considering that the flow rules shows $\dot{\omega}=\dot{\lambda}_{d}>0$ only if $\phi_{d}=0$, which considering the first of Eq.(17) gives

$$
D_{d}=Y \dot{\omega}-\chi \dot{\xi}=\left(\frac{1}{2} A_{n} u_{n}^{2}+\frac{1}{2} A_{t} u_{t}^{2}+Y_{0}\right) \dot{\lambda}_{d} \geq 0
$$

showing the unconditioned positiveness of the dissipation rate for any damage increment, being $D_{d}=0$ only if $\dot{\lambda}_{d}=0$.

Finally, in order to prevent damage activation under pure compressive stress state, normal stiffness of the sound fraction and normal stiffness of the cracked fraction are imposed to be equal, that is $K_{n}^{s}=K_{n}^{c}$. In fact, for a displacement 
and by means of the following plastic potential

$$
\Omega_{p}\left(\boldsymbol{t}_{c}\right)=\left|t_{c t}\right|+\beta t_{c n} \leq 0
$$

271 where $t_{c n}$ and $t_{c t}$ are the normal and tangential components of the traction ${ }_{272}$ vector $\boldsymbol{t}_{c} ; \alpha$ and $\beta$, with $\alpha \geq \beta$ are the frictional and the dilatancy coefficients 274 conditions read

$$
\begin{aligned}
\dot{\delta}_{n}^{p} & =\frac{\partial \Omega_{p}}{\partial t_{c n}} \dot{\lambda}_{p}=\operatorname{sgn}\left(t_{c t}\right) \dot{\lambda}_{p}, \\
\dot{\delta}_{t}^{p} & =\frac{\partial \Omega_{p}}{\partial t_{c t}} \dot{\lambda}_{p}=\beta \dot{\lambda}_{p}, \\
\dot{\lambda}_{p} & \geq 0, \quad \phi_{p} \dot{\lambda}_{p}=0, \quad \dot{\phi}_{p} \dot{\lambda}_{p}=0
\end{aligned}
$$

275

The dissipation rate associated with frictional active mechanisms is evaluated considering that $\dot{\lambda}_{p}>0$ only if $\phi_{p}=0$ which, considering the second of the Eqs.(17), gives

$$
D_{p}=\boldsymbol{t}_{c}^{T} \dot{\boldsymbol{\delta}}^{p}=\left(\left|t_{c t}\right|+\beta t_{c n}\right) \dot{\lambda}_{p} \geq\left(\left|t_{c t}\right|+\alpha t_{c n}\right) \dot{\lambda}_{p}=0
$$

which shows that dissipation is always positive for any frictional rate displacement rate, with $D_{p}=0$ only if $\dot{\lambda}_{p}=0$

The cohesive model is then completed by the state laws for the internal variable $\chi$ which drive the damage evolution law. In case of simple linear softening 
in normal traction-opening displacement (mode I), it can be shown that internal free energy and internal state law read:

$$
\begin{array}{r}
\psi_{i}(\xi)=G_{I} \frac{u_{e}}{u_{f}}\left(\frac{u_{f}^{2} /\left(u_{f}-u_{e}\right)}{u_{f}(1-\xi)+u_{e} \xi}-\xi\right) \\
\chi(\xi):=\frac{d \psi_{i}(\xi)}{d \xi}=G_{I} \frac{u_{e}}{u_{f}}\left[\left(\frac{u_{f}}{u_{f}(1-\xi)+u_{e} \xi}\right)^{2}-1\right]
\end{array}
$$

where $u_{e}$ and $u_{f}$ are the separation displacements for the limit elastic threshold and for the full damage condition ( $\omega=1$, i.e. full detachment) in pure mode I opening condition, $G_{I}=1 / 2 K_{n}^{s} u_{e} u_{f}$ is the fracture energy in mode I and finally, $K_{n}^{s}$ is the stiffness normal component of the interface sound fraction. As far as the other material parameters are concerned, it is set

$$
\begin{aligned}
Y_{0} & =\frac{1}{2} K_{n}^{s} u_{e}^{2} \equiv G_{I} \frac{u_{e}}{u_{f}} \\
A_{t} & =K_{t}^{s}\left(1-\frac{G_{I}}{G_{I I}}\right) ; \quad A_{n}=0
\end{aligned}
$$

The two constants $A_{n}$ and $A_{t}$ have been fixed under the condition that the fracture energy in mode II $\left(G_{I I}\right)$ is greater than the fracture energy in mode I $\left(G_{I}\right)$, i.e. $G_{I I}>G_{I}$. Finally, the relations in Eqs.(28) among the fracture energies and the parameters $Y_{0}$ and $A_{t}$ will be explained in detail in Sect. 3 .

\section{Monotonic loading paths}

In this Section the monotonic mixed delamination path, represented in Figure 2 for a linear interface element, is analyzed. The displacement jump $\boldsymbol{u}$ is decomposed in the local Cartesian components as

$$
\boldsymbol{u}=u_{t} \boldsymbol{e}_{t}+u_{n} \boldsymbol{e}_{n}=u \cos \gamma \boldsymbol{e}_{t}+u \sin \gamma \boldsymbol{e}_{n}
$$

where $u=\left(\boldsymbol{u}^{T} \boldsymbol{u}\right)^{1 / 2}$ and $\boldsymbol{e}_{t}$, and $\boldsymbol{e}_{n}$ are the unit tangential and normal vectors to the interface plane.

The pure mode I delamination condition is produced by a loading angle $\gamma=\pi / 2$ and the pure mode II delamination condition is obtained by assuming a loading angle $\gamma=0$. 
Figure 2: Monotonic mixed mode loading scheme. The displacement jump $\boldsymbol{u}$ decomposed as $\boldsymbol{u}=u_{t} \boldsymbol{e}_{t}+u_{n} \boldsymbol{e}_{n}$.

The separation work $W=W_{n}+W_{t}$ can be defined as the sum of two different contributions, namely: the normal separation work $W_{n}$ and the tangential one $W_{t}$, which are mathematically defined as

$$
\begin{aligned}
W_{n} & =\int_{0}^{+\infty} t_{n}(\gamma, \boldsymbol{u}) \mathrm{d} u_{n} \\
W_{t} & =\int_{0}^{+\infty} t_{t}(\gamma, \boldsymbol{u}) \mathrm{d} u_{t}
\end{aligned}
$$

Due to the assumed non-negative opening displacement $\left(u_{n} \geq 0\right)$, the frictional Traction components are

$$
\begin{aligned}
t_{n} & =(1-\omega) K_{n}^{s} u \sin (\gamma) \\
t_{t} & =(1-\omega) K_{t}^{s} u \cos (\gamma)
\end{aligned}
$$

31 The initial interface behavior is elastic with null damage and null internal vari312 able $(\omega=0$ and $\xi=0)$ and the maximum elastic traction is reached when 313 the damage activation condition is attained $\phi_{d}\left(Y, u_{t}, \chi\right)=0$, where $\chi(0)=0$, ${ }_{314} A_{n}=0, A_{t}>0$ and the energy release rate is

$$
Y=\frac{1}{2} K_{n}^{s} u^{2} \sin ^{2}(\gamma)+\frac{1}{2} K_{t}^{s} u^{2} \cos ^{2}(\gamma)
$$


therefore

$$
\begin{aligned}
\phi_{d}\left(Y, u_{t}, \chi\right) & =Y-\chi(\xi)-\frac{1}{2} A_{t} u_{t}^{2}-Y_{0}= \\
& =\frac{1}{2} K_{n}^{s} u^{2} \sin ^{2}(\gamma)+\frac{1}{2}\left(K_{t}^{s}-A_{t}\right) u^{2} \cos ^{2}(\gamma)-Y_{0}= \\
& =\frac{1}{2} \frac{K_{n}^{s} u^{2}}{C^{2}(\gamma)}-Y_{0}=0
\end{aligned}
$$

316 where

$$
C(\gamma)=\left(\sin ^{2}(\gamma)+\frac{K_{t}^{s}-A_{t}}{K_{n}^{s}} \cos ^{2}(\gamma)\right)^{-1 / 2}
$$

317 is a loading angle dependent function. For $\gamma=\pi / 2$ (opening in mode I) $318 C(\pi / 2)=1$, whereas for $\gamma=0$ (sliding in mode II) $C(0)=\sqrt{K_{n}^{s} /\left(K_{t}^{s}-A_{t}\right)}$.

By substitution of the first of Eqs. (28) in Eq. (33), the imposed separation 320 displacement $\bar{u}_{e}$ at the limit elastic is

$$
\bar{u}_{e}=u_{e} C(\gamma)
$$

321 The linear-elastic branch is followed by a descending (softening) one with in322 creasing damage and, in virtue of flow rules of Eqs. (20), damage activation 323 function (18) and softening law (27), kinematic internal variable and damage 324 variable are

$$
\xi=\omega=\frac{u_{f}}{u_{f}-u_{e}}\left[1-\frac{u_{e}}{u} C(\gamma)\right]
$$

325 and separation displacement $\bar{u}_{f}$ at the fully damaged condition $(\omega=1)$ is

$$
\bar{u}_{f}=u_{f} C(\gamma)
$$

326 The traction components at the descending branch are obtained from Eqs. (31) 327 and (36)

$$
\begin{aligned}
t_{n}(u, \gamma) & =\frac{u_{f} u_{e} C(\gamma)-u_{e} u}{u_{f}-u_{e}} K_{n}^{s} \sin (\gamma) \\
t_{t}(u, \gamma) & =\frac{u_{f} u_{e} C(\gamma)-u_{e} u}{u_{f}-u_{e}} K_{t}^{s} \cos (\gamma)
\end{aligned}
$$

328 The work done by normal traction and the work done by tangential one can be 329 computed by Eqs. (30) and are

$$
\begin{aligned}
W_{n}(\gamma) & =\frac{1}{2} K_{n}^{s} u_{e} u_{f} C^{2}(\gamma) \sin ^{2}(\gamma), \\
W_{t}(\gamma) & =\frac{1}{2} K_{t}^{s} u_{e} u_{f} C^{2}(\gamma) \cos ^{2}(\gamma) .
\end{aligned}
$$


In Figure (3) the qualitatively response of the interface subjected to the mono-

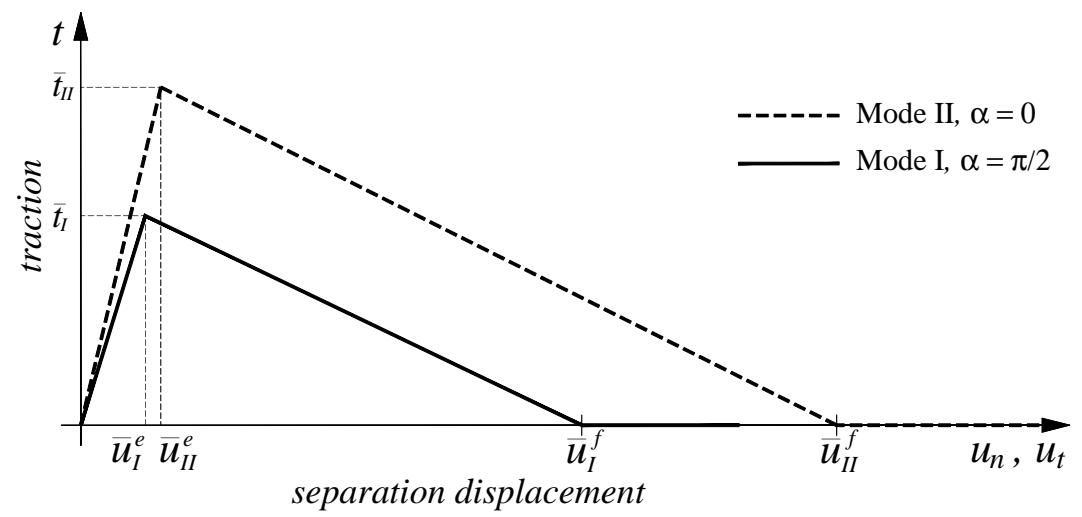

Figure 3: Mode I response and mode II response for monotonic loading path.

330

331 tonic loading path is represented in terms of traction $v s$ separation displacement for the two limit cases of pure mode I and pure mode II.

333

In the pure mode I debonding condition $(\gamma=\pi / 2)$ limit elastic displacement ${ }_{334} \bar{u}_{I}^{e}$, the fully debonding displacement $\bar{u}_{I}^{f}$ and the maximum normal traction $\bar{t}_{I}$, 335 respectively are

$$
\begin{aligned}
& \bar{u}_{I}^{e}=u_{e} \\
& \bar{u}_{I}^{f}=u_{f} \\
& \bar{t}_{I}=K_{n}^{s} u_{e},
\end{aligned}
$$

336 whereas, in the pure mode II debonding condition $(\gamma=0)$ limit elastic displace337 ment $\bar{u}_{I I}^{e}$, fully debonding displacement $\bar{u}_{I I}^{f}$ and maximum normal traction $\bar{t}_{I I}$, 
respectively are

$$
\begin{aligned}
& \bar{u}_{I I}^{e}=\sqrt{\frac{K_{n}^{s}}{K_{t}^{s}-A_{t}}} u_{e} \\
& \bar{u}_{I I}^{f}=\sqrt{\frac{K_{n}^{s}}{K_{t}^{s}-A_{t}}} u_{f} \\
& \bar{t}_{I I}=\sqrt{\frac{K_{n}^{s}}{K_{t}^{s}-A_{t}}} K_{t}^{s} u_{e}
\end{aligned}
$$

34 whereas the tangential separation work is $W_{t}(\pi / 2)=0$. The mode II fracture 342 energy is given by the tangential separation work for $\gamma=0$, that is

$$
G_{I I}=W_{t}(0)=\frac{1}{2} K_{n}^{s} u_{e} u_{f} \frac{K_{t}^{s}}{K_{t}^{s}-A_{t}}=G_{I} \frac{K_{t}^{s}}{K_{t}^{s}-A_{t}}
$$

343 and the normal separation work is $W_{n}(0)=0$. Equation (43) confirms that for ${ }_{344} A_{t}>0$ mode II fracture energy is greater the the mode I value, $G_{I I}>G_{I}$.

345 It can also be shown from Eqs. (39) that

$$
W_{n}(\gamma)=G_{I} C^{2}(\gamma) \sin ^{2}(\gamma) ; \quad W_{t}(\gamma)=G_{I} \frac{K_{t}^{s}}{K_{n}^{s}} C^{2}(\gamma) \cos ^{2}(\gamma)
$$

346 and then

$$
W(\gamma)=W_{n}(\gamma)+W_{t}(\gamma)=G_{I} C^{2}(\gamma)\left[\sin ^{2}(\gamma)+\frac{K_{t}^{s}}{K_{n}^{s}} \cos ^{2}(\gamma)\right]
$$

347 In Figures 4 the work of separation for the monotonic loading path is qual348 itatively represented as function of angle $\gamma$, where it can be observed that, for 349 any mixed mode debonding condition, the separation work is

$$
G_{I} \leq W(\alpha) \leq G_{I I}
$$

350 and it monotonically increases from the pure mode I condition to the pure mode

351 II condition. Several experimental investigations confirm that fracture energy 
352

in mixed mode debonding condition gradually and monotonically increases from the pure mode I value $G_{I}$ to the pure mode II value $G_{I I}$. Such a result is reported by Benzeggagh and Kenane (1996), who measured the fracture energy of a unidirectional glass/epoxy composite for six different mixed mode conditions, by the mixed mode bending apparatus developed by Crews and Reeder (1998)

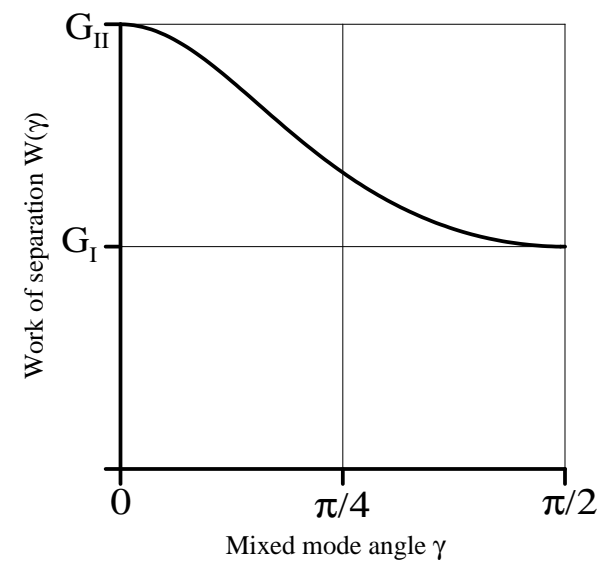

Figure 4: Work of separation in the monotonic loading path, in function of delamination angle $\gamma$.

\section{Non-proportional loading paths}

The behaviour of the proposed model is also analyzed for two non-proportional loading paths, well known in literature (van den Bosch et al., 2006; Park et al., 2009; Dimitri et al., 2014) for the validation of debonding models with different fracture energies in mode I and in mode II.

The first non-proportional loading path (a) is applied by an initial opening displacement, which increases up to a maximum value $u_{n}=u_{a 1}$, and by a subsequent sliding displacement $u_{t}=u_{a 2}$, which increases up to complete delamination.

The second non-proportional loading path (b) is applied by an initial sliding displacement, which increases up to a maximum value $u_{t}=u_{b 1}$, and by 


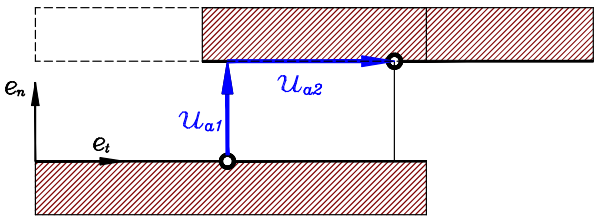

a) tively in Fig.(5a) and in Fig.(5b).

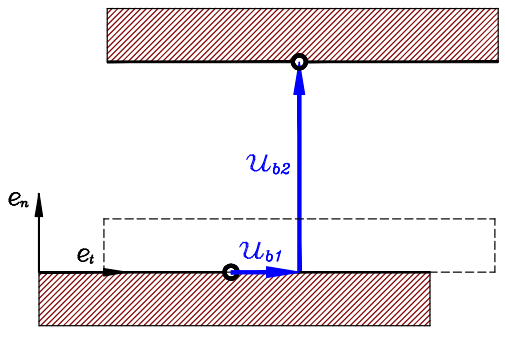

b)

Figure 5: Non-proportional loading paths: a) path (a) opening displacement and subsequent

sliding displacement; b) path (b) sliding displacement and subsequent opening displacement.

370

a subsequent opening displacement $u_{n}=u_{b 2}$, which increases up to complete

\subsection{Non-proportional loading path (a)}

The solution of non-proportional loading can be developed analytically and two different cases has to be distinguished:

- the initial normal displacement is less than or equal to the mode I limit elastic displacement $\left(u_{a 1} \leq \bar{u}_{I}^{e}=u_{e}\right)$;

- the initial normal displacement is greater than or equal to the mode I limit elastic displacement and less than the fully debonding displacement $\left(\bar{u}_{I}^{e} \leq u_{a 1}<\bar{u}_{I}^{f}\right)$.

In the first case, the normal displacement $u_{n}=u_{a 1}$ produces elastic response and the second loading branch, with tangential displacement $u_{t}=u_{a 2}$, is initially elastic. The first damage activation is reached at following tangential displacement

$$
\bar{u}_{t}^{e}=\left[\frac{K_{n}^{s}}{K_{t}^{s}-A_{t}}\left(u_{e}^{2}-u_{a 1}^{2}\right)\right]^{\frac{1}{2}}
$$



mode I elastic limit value $\left(u_{n}^{a_{1}} \leq \bar{u}_{I}^{e}=u_{e}\right)$.

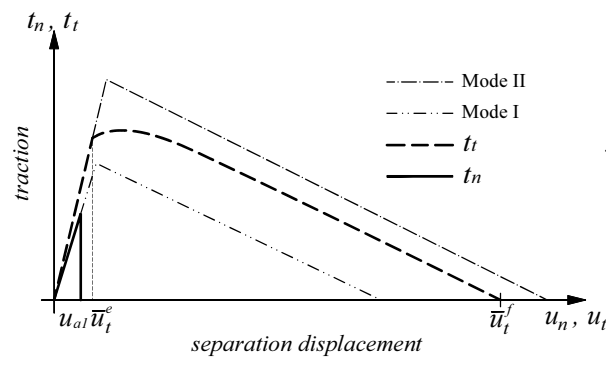

a)

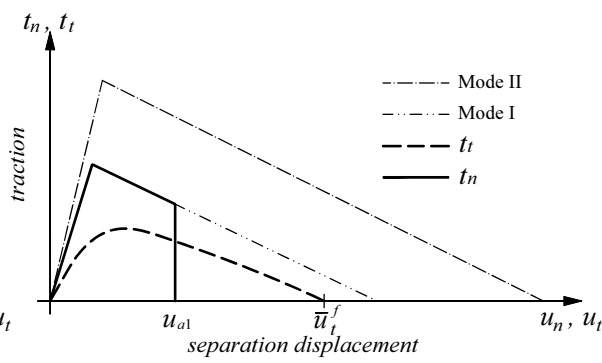

b)

Figure 6: Non-proportional loading path (a): a) initial normal displacement less than the mode I elastic limit value $\left.\left(u_{a 1} \leq \bar{u}_{I}^{e}\right) ; \mathrm{b}\right)$ initial normal displacement greater than the mode I elastic limit value $\left(\bar{u}_{I}^{e} \leq u_{a 1}<\bar{u}_{I}^{f}\right)$.

$$
\bar{u}_{t}^{f}=\left[\frac{K_{n}^{s}}{K_{t}^{s}-A_{t}}\left(u_{f}^{2}-u_{a 1}^{2}\right)\right]^{\frac{1}{2}} .
$$

$$
\begin{aligned}
& t_{n}\left(u_{a 1}, u_{a 2}\right)=-\frac{u_{e}}{u_{f}-u_{e}} K_{n}^{s} u_{a 1}+\frac{u_{f}}{u_{f}-u_{e}} K_{n}^{s} u_{e} u_{a 1}\left[u_{a 1}^{2}+\frac{K_{t}^{s}-A_{t}}{K_{n}^{s}} u_{a 2}^{2}\right]^{-1 / 2} \\
& t_{t}\left(u_{a 1}, u_{a 2}\right)=-\frac{u_{e}}{u_{f}-u_{e}} K_{t}^{s} u_{a 2}+\frac{u_{f}}{u_{f}-u_{e}} K_{t}^{s} u_{e} u_{a 2}\left[u_{a 1}^{2}+\frac{K_{t}^{s}-A_{t}}{K_{n}^{s}} u_{a 2}^{2}\right]^{-1 / 2}
\end{aligned}
$$

The qualitatively behaviour of the proposed model, in terms of traction mponents vs separation displacements, for the non-proportional loading path (a) is represented in Fig.(6a) for an initial normal displacement less than the

Finally, the work done by normal traction, the work done by tangential traction and total work-of-separation, respectively, are

$$
\begin{aligned}
W_{n} & =\frac{1}{2} K_{n}^{s} u_{a 1}^{2} \\
W_{t} & =\frac{1}{2} K_{t}^{s} \bar{u}_{t}^{e}{ }^{2}+\int_{\bar{u}_{t}^{e}}^{\bar{u}_{t}^{f}} t_{t} \mathrm{~d} u_{t}=G_{I I}-\frac{K_{t}^{s}}{K_{t}^{s}-A_{t}} \frac{1}{2} K_{n}^{s} u_{a 1}^{2} \\
W & =W_{n}+W_{t}=G_{I I}-\frac{A_{t}}{K_{t}^{s}-A_{t}} \frac{1}{2} K_{n}^{s} u_{a 1}^{2} .
\end{aligned}
$$


The second case of non-proportional loading path (a) is obtained with an initial normal displacement greater than or equal to the mode I limit elastic value and less than the fully debonding displacement $\left(\bar{u}_{I}^{e} \leq u_{a 1}<\bar{u}_{I}^{f}\right)$. The damage value at the end of the first loading branch $\left(u_{n}=u_{a 1}, u_{t}=0\right)$ is

$$
\omega\left(u_{a 1}, 0\right)=\frac{u_{f}}{u_{f}-u_{e}} \frac{u_{a 1}-u_{e}}{u_{a 1}}
$$

and the relevant traction components are

$$
\begin{aligned}
& t_{n}\left(u_{a 1}, 0\right)=k_{n}^{s} u_{e} \frac{u_{f}-u_{a 1}}{u_{f}-u_{e}} \\
& t_{t}\left(u_{a 1}, 0\right)=0 .
\end{aligned}
$$

The behaviour in the second loading branch $\left(u_{n}=u_{a 1}, u_{t}=u_{a 2}\right)$ is completely nonlinear and the traction components are given by Eqs. (49a, b). The tangential displacement at the fully debonded condition is again given by Eq. (48), obtained for the first case.

The qualitatively behaviour of the proposed model, in terms of traction components vs separation displacements, for the non-proportional loading path (a) is represented in Figure (6b) for an initial normal displacement greater than the mode I elastic limit value $\left(\bar{u}_{I}^{e} \leq u_{a 1}<\bar{u}_{I}^{f}\right)$.

Finally, the work done by normal traction, the work done by tangential traction and total work-of-separation, respectively, are

$$
\begin{aligned}
W_{n} & =\int_{0}^{u_{a 1}} t_{n} d u_{n}=G_{I}-G_{I} \frac{\left(u_{f}-u_{a 1}\right)^{2}}{u_{f}\left(u_{f}-u_{e}\right)} \\
W_{t} & =\int_{0}^{\bar{u}_{t}^{f}} t_{t} d u_{t}=G_{I I} \frac{\left(u_{f}-u_{a 1}\right)^{2}}{u_{f}\left(u_{f}-u_{e}\right)} \\
W & =W_{n}+W_{t}=G_{I}+\left(G_{I I}-G_{I}\right) \frac{\left(u_{f}-u_{a 1}\right)^{2}}{u_{f}\left(u_{f}-u_{e}\right)} .
\end{aligned}
$$

\subsection{Non-proportional loading path (b)}

The non-proportional loading path (b) is schematically represented in Fig.(5b) and it imposes an initial tangential displacement $u_{b 1}$ and than a monotonically increasing normal displacement $u_{b 2}$ is applied up to the fully debonding.

Similar to the previous loading path (a), the analytical solution of the loading path (b) has to be developed for two different cases: 
- the initial tangential displacement is less than or equal to the mode II limit elastic displacement $\left(u_{b 1} \leq \bar{u}_{I I}^{e}\right)$;

- the initial tangential displacement is greater than or equal to the mode II limit elastic displacement and less than the fully debonding displacement $\left(\bar{u}_{I I}^{e} \leq u_{b 1}<\bar{u}_{I I}^{f}\right)$.

In the first case, the tangential displacement $u_{b 1}$ produces elastic response and the second loading branch, with normal displacement $u_{b 2}$, is initially elastic. The first damage activation is reached at following normal displacement

$$
\bar{u}_{n}^{e}=\left[\bar{u}_{I}^{e} 2-\frac{K_{t}^{s}-A_{t}}{K_{n}^{s}} u_{b 1}^{2}\right]^{\frac{1}{2}}
$$

while the fully debonding condition $(\omega=1)$ is reached at normal displacement

$$
\bar{u}_{n}^{f}=\left[\bar{u}_{I}^{f 2}-\frac{K_{t}^{s}-A_{t}}{K_{n}^{s}} u_{b 1}^{2}\right]^{\frac{1}{2}} .
$$

¿22 Traction components after damage activation, for $\bar{u}_{n}^{e} \leq u_{b 2} \leq \bar{u}_{n}^{f}$, are

$$
\begin{aligned}
t_{n}\left(u_{b 2}, u_{b 1}\right) & =-\frac{u_{e}}{u_{f}-u_{e}} K_{n}^{s} u_{b 2}+\frac{u_{f}}{u_{f}-u_{e}} K_{n}^{s} u_{b 2} u_{e}\left[u_{b 2}^{2}+\frac{K_{t}^{s}-A_{t}}{K_{n}^{s}} u_{b 1}^{2}\right]^{-\frac{1}{2}} \\
t_{t}\left(u_{b 2}, u_{b 1}\right) & =-\frac{u_{e}}{u_{f}-u_{e}} K_{t}^{s} u_{b 1}+\frac{u_{f}}{u_{f}-u_{e}} K_{t}^{s} u_{b 1} u_{e}\left[u_{b 2}^{2}+\frac{K_{t}^{s}-A_{t}}{K_{n}^{s}} u_{b 1}^{2}\right]^{-\frac{1}{2}}
\end{aligned}
$$

The qualitatively behaviour of the proposed model, in terms of traction components vs separation displacements, for the non-proportional loading path (b) is represented in Figure (7a) for an initial tangential displacement less than the mode II elastic limit value $\left(u_{b 1} \leq \bar{u}_{I I}^{e}\right)$.

Finally, the work done by normal traction, the work done by the tangential one and the total work-of-separation, respectively, are

$$
\begin{aligned}
W_{n} & =\frac{1}{2} K_{n}^{s} \bar{u}_{n}^{e 2}+\int_{\bar{u}_{n}^{e}}^{\bar{u}_{n}^{f}} t_{n} \mathrm{~d} u_{n}=G_{I}-\frac{1}{2}\left(K_{t}^{s}-A_{t}\right) u_{b 1}^{2} \\
W_{t} & =\frac{1}{2} K_{t}^{s} u_{b 1}^{2} \\
W & =W_{n}+W_{t}=G_{I}+\frac{1}{2} A_{t} u_{b 1}^{2} .
\end{aligned}
$$

The second case of non-proportional loading path (b) is obtained with an initial tangential displacement greater than or equal to the mode II limit elastic 


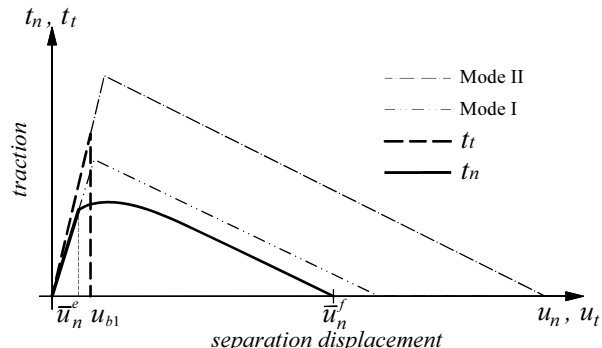

a)

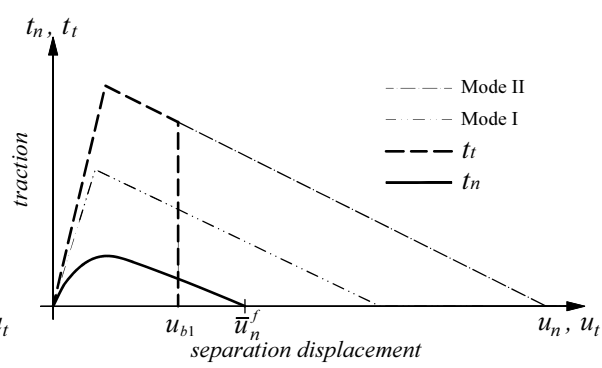

b)

Figure 7: Non-proportional loading path (b): a) initial tangential displacement less than the mode II elastic limit value $\left.\left(u_{b 1} \leq \bar{u}_{I I}^{e}\right) ; \mathrm{b}\right)$ initial tangential displacement greater than the mode II elastic limit value $\left(\bar{u}_{I I}^{e} \leq u_{b 1}<\bar{u}_{I I}^{f}\right)$.

${ }_{431}$ value and less than the fully debonding displacement $\left(\bar{u}_{I I}^{e} \leq u_{b 1}<\bar{u}_{I I}^{f}\right)$. The

${ }_{432}$ damage value at the end of the first loading branch $\left(u_{n}=0, u_{t}=u_{b 1}\right)$ is

$$
\omega\left(0, u_{b 1}\right)=\frac{u_{f}}{u_{f}-u_{e}} \frac{u_{b 1}-\bar{u}_{I I}^{e}}{u_{b 1}}
$$

${ }_{433}$ and the relevant traction components are

$$
\begin{aligned}
& t_{n}\left(0, u_{b 1}\right)=0 \\
& t_{t}\left(0, u_{b 1}\right)=K_{t}^{s} \bar{u}_{I I}^{e} \frac{\bar{u}_{I I}^{f}-u_{b 1}}{\bar{u}_{I I}^{f}-\bar{u}_{I I}^{e}} .
\end{aligned}
$$

${ }_{434}$ The behavior in the second loading branch $\left(u_{n}=u_{b 2}, u_{t}=u_{b 1}\right)$ is completely ${ }_{435}$ nonlinear and the traction components are defined by the same relations of ${ }_{436}$ previous case, that are given by Eqs. $(56 \mathrm{a}, \mathrm{b})$. The tangential displacement at ${ }_{437}$ the fully debonded condition is again given by Eq. (55), obtained for the first 438 case.

${ }_{439}$ The qualitatively behavior of the proposed model, in terms of traction com${ }_{440}$ ponents $v s$ separation displacements, for the non-proportional loading path (b)

$44 \mathbf{1}$ is represented in Figure (7b) for an initial tangential displacement greater than

442 the mode II elastic limit value $\left(\bar{u}_{I I}^{e} \leq u_{b 1}<\bar{u}_{I I}^{f}\right)$.

${ }_{443}$ Finally, the work done by normal traction, the work done by tangential 
${ }_{444}$ traction and total work-of-separation, respectively, are

$$
\begin{aligned}
& W_{n}=\int_{0}^{\bar{u}_{n}^{f}} t_{n} \mathrm{~d} u_{n}=G_{I} \frac{\left(\bar{u}_{I I}^{f}-u_{b 1}\right)^{2}}{\bar{u}_{I I}^{f}\left(\bar{u}_{I I}^{f}-\bar{u}_{I I}^{e}\right)} \\
& W_{t}=\int_{0}^{\bar{u}_{t}^{f}} t_{t} \mathrm{~d} u_{t}=G_{I I}-G_{I I} \frac{\left(\bar{u}_{I I}^{f}-u_{b 1}\right)^{2}}{\bar{u}_{I I}^{f}\left(\bar{u}_{I I}^{f}-\bar{u}_{I I}^{e}\right)} \\
& W=W_{n}+W_{t}=G_{I I}+\left(G_{I}-G_{I I}\right) \frac{\left(\bar{u}_{I I}^{f}-u_{b 1}\right)^{2}}{\bar{u}_{I I}^{f}\left(\bar{u}_{I I}^{f}-\bar{u}_{I I}^{e}\right)} .
\end{aligned}
$$

${ }_{445}$ The work done by the normal traction, the work done by the tangential one and

446 the total work-of-separation, performed in the non-proportional loading path

${ }_{447}$ (a), are plotted in Figure (8) in function of the initial normal displacement $u_{a 1}$.

${ }_{448}$ The work done by the normal traction, the work done by the tangential one and

${ }_{449}$ the total work-of-separation, performed in the non-proportional loading path

${ }_{450}$ (b), are plotted in Figure. (9) in function of the initial tangential displacement

${ }_{451} u_{b 1}$.

452 The results plotted in the Figs. (8) and (9) have been evaluated with the

${ }_{453}$ following constitutive parameters: $k_{n}^{s}=k_{t}^{s}=1000 \mathrm{~N} / \mathrm{mm}^{3}, A_{t}=500 \mathrm{~N} / \mathrm{mm}^{3}$,

${ }_{454} u_{e}=0.005 \mathrm{~mm}, u_{f}=0.04 \mathrm{~mm}$; which produces the mode I fracture energy $G_{I}=$

${ }_{455} 0.1 \mathrm{~N} / \mathrm{mm}=100 \mathrm{~J} / \mathrm{m}^{2}$ and the mode II fracture energy $G_{I I}=0.2 \mathrm{~N} / \mathrm{mm}=$

${ }_{456} 200 \mathrm{~J} / \mathrm{m}^{2}$. Moreover, displacements at the initial damage condition and at

${ }_{457}$ the fully debonded one, in pure mode I loading law, are: $\bar{u}_{I}^{e}=0.005 \mathrm{~mm}$,

${ }_{458} \bar{u}_{I}^{f}=0.04 \mathrm{~mm}$; and in pure mode II loading law: $\bar{u}_{I I}^{e}=0.00707 \mathrm{~mm}, \bar{u}_{I I}^{f}=$

$4590.05657 \mathrm{~mm}$.

${ }_{460}$ The graphs in Figures (8) and (9) show the path dependency of work-of-

${ }_{461}$ separation and, especially, its smooth and monotonic transition from the mode

462 I fracture energy $G_{I}$ to the mode II fracture energy $G_{I I}$ and vice versa.

${ }_{463}$ For the first non-proportional loading path (Figure 5 a), a null initial nor-

${ }_{464}$ mal displacement $u_{a 1}=0$ produces a pure mode II failure and, as shown in

${ }_{465}$ Figure (8), the total work-of-separation is $W=W_{t}=G_{I I}$ and work done by

${ }_{466}$ normal traction is $W_{n}=0$. On the contrary, the initial normal displacement 


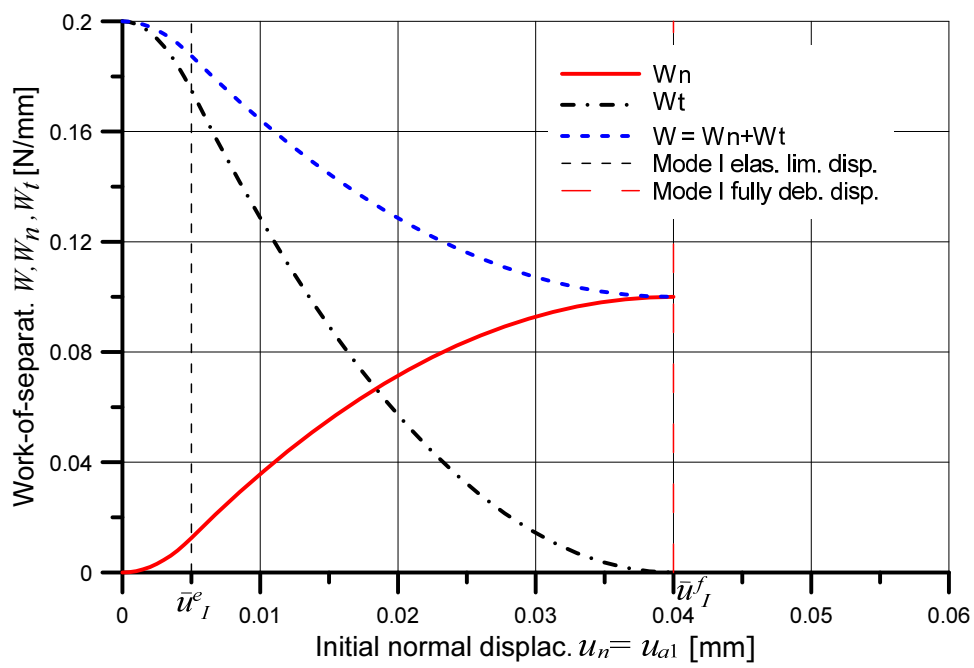

Figure 8: Work done by the normal traction, work done by the tangential traction and the total work-of-separation, performed in the non-proportional loading path (a).

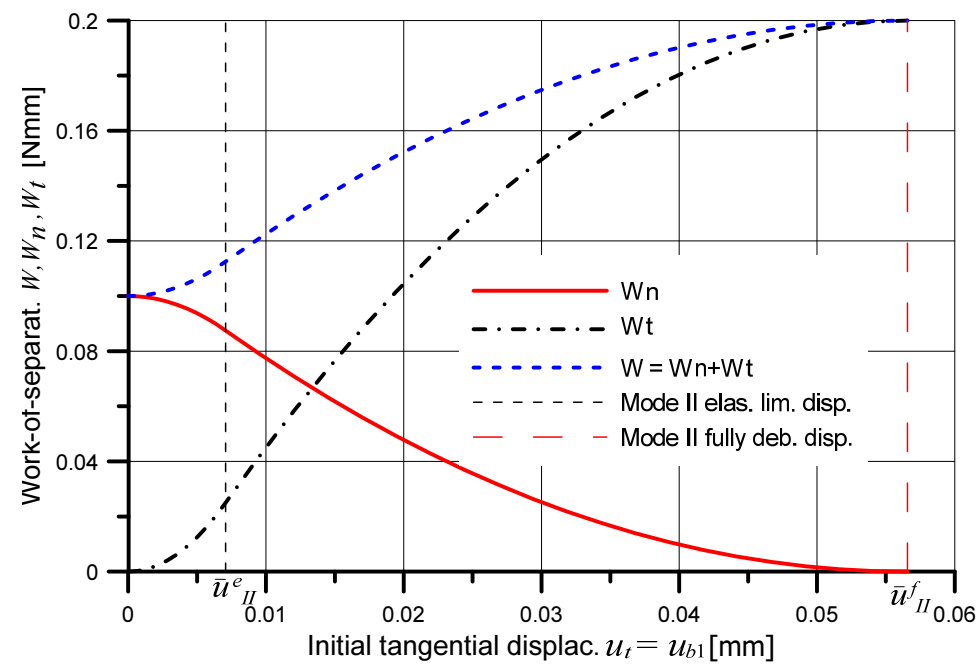

Figure 9: Work done by the normal traction, work done by the tangential traction and the total work-of-separation, performed in the non-proportional loading path (b). 
${ }_{467} u_{a 1}=\bar{u}_{I}^{f}$ produces a pure mode I failure and the total work-of-separation is ${ }_{468} W=W_{n}=G_{I}$ and work done by tangential traction is $W_{t}=0$, as con${ }_{469}$ firmed in Figure (8). In Figure (8), the other values of the normal displacement ${ }_{470} 0<u_{a 1}<\bar{u}_{I}^{f}$ produce mixed mode failure conditions with smooth and mono471 tonic variation of normal work $W_{n}$, tangential work $W_{t}$ and total work $W$ from 472 the pure mode II condition to the pure mode I condition.

Analogous results can be observed in Figure (9) for the second non-proportional ${ }_{474}$ loading path (Figure $5 \mathrm{~b}$ ), where an initial tangential displacement $u_{b 1}=0$ pro${ }_{475}$ duces a pure mode I failure and $u_{b 1}=\bar{u}_{I I}^{f}$ produces a pure mode II failure.

476 In Dimitri et al. (2014) the response to the non-proportional loading paths 477 (a) and (b) of some interface constitutive models (van den Bosch et al., 2006; 478 McGarry et al., 2014; Högberg, 2006; Camanho et al., 2003) are reported in terms of total work-of-separation, work done by normal traction and work done

${ }_{480}$ by tangential traction, as function of the ratios $u_{a 1} / \bar{u}_{I}^{f}$ for the path (a) and as ${ }_{481}$ function of the ratios $u_{b 1} / \bar{u}_{I I}^{f}$ for the path (b).

In order to compare the proposed model with models available in literature, 483 the results of the two non-proportional loading paths (a) and (b) plotted in ${ }_{484}$ Figures (8) and (9) are based on the same values of mode I fracture energy $G_{I}$ 485 and mode II fracture energy $G_{I I}$ assumed in Dimitri et al. (2014).

Several CZMs proposed in literature (Xu and Needleman, 1993; Högberg, 2006; Camanho et al., 2003) are inaccurate in mixed mode failure conditions (see Dimitri et al. (2014) for a comparative analysis), producing work-of-separation less than mode I fracture energy $\left(W<G_{I}\right)$ or greater than mode II fracture 490 energy $\left(W>G_{I I}\right)$. On the contrary, the CZMs proposed in van den Bosch et al. ${ }_{491}$ (2006); Dimitri et al. (2014); Park et al. (2009) produce normal work, tangential 492 work and total work-of-separation qualitatively similar to the results plotted 493 respectively, in Figure (8) for the first non-proportional loading path and in ${ }_{494}$ Fig.(9) for the second non-proportional loading path. However, CMZs proposed 495 in van den Bosch et al. (2006); Park et al. (2009) are not based on an Helmholtz 496 free energy and are not thermodynamically consistent; the CZM proposed in ${ }_{497}$ Dimitri et al. (2014) is fully thermodynamically consistent, but it is based on 
four scalar damage variable, whose physical or mechanical interpretation is not evident. Moreover, such model does not allows to consider frictional effects on the damaged fraction, by the mesoscale interpretation proposed in Parrinello et al. (2009) and Alfano and Sacco (2006).

\section{Numerical simulation}

The proposed model has been implemented in the finite element code FEAP (Zienkiewicz and Taylor, 2000) and three different delamination tests have been numerically simulated, namely:

- end-notched double cantilever beam test;

- a mixed mode bending test on end-notched specimen.

- a four points end-notched flexural delamination test;

The numerical simulations have been performed using 2D nine nodes plane stress elements and six nodes interface elements. The bulk is modeled as isotropic and linear elastic with Young modulus $E=35300 \mathrm{~N} / \mathrm{mm}^{2}$ and Poisson ratio $\nu=0.3$ (standard parameters for E-glass/epoxy composite material). Two different sets of interface constitutive parameters have been considered, both with the same fracture energies $\left(G_{I}=1 \mathrm{~N} / \mathrm{mm}\right.$ and $\left.G_{I I}=4 \mathrm{~N} / \mathrm{mm}\right)$ but with different normal tensile strength and shear strength. The first set is reported in Table 1 and produces normal tensile strength $\bar{t}_{I}=20 \mathrm{~N} / \mathrm{mm}^{2}$ and shear strength $\bar{t}_{I I}=40 \mathrm{~N} / \mathrm{mm}^{2}$, whereas the second set of constitutive parameters produces normal tensile strength $\bar{t}_{I}=40 \mathrm{~N} / \mathrm{mm}^{2}$ and shear strength $\bar{t}_{I I}=80 \mathrm{~N} / \mathrm{mm}^{2}$

The analytical solutions of the three delamination tests are known in literature and developed in the framework of classical linear elastic fracture mechanics coupled with bending beam theory.

\subsection{DCB test}

Sizes and geometry of analyzed specimen are represented in Fig.10 and the analytical response, under bending beam theory and linear fracture mechanics 


\begin{tabular}{|l|l|}
\hline \hline & Cohesive Parameters \\
\hline Normal elastic stiffness & $K_{n}^{s}=50000 \mathrm{~N} / \mathrm{mm}^{3}$ \\
Tangential elastic stiffness & $K_{t}^{s}=50000 \mathrm{~N} / \mathrm{mm}^{3}$ \\
Mixed mode parameter & $A_{t}=37500 \mathrm{~N} / \mathrm{mm}^{3}$ \\
Mode I elastic displ. & $\bar{u}_{I}^{e}=u_{e}=0.0004 \mathrm{~mm}$ \\
Mode I debonding displ. & $\bar{u}_{I}^{f}=u_{f}=0.1 \mathrm{~mm}$ \\
Tensile strength & $\bar{t}_{I}=20 \mathrm{~N} / \mathrm{mm}^{2}$ \\
Mode II elastic displ. & $\bar{u}_{I I}^{e}=0.0008 \mathrm{~mm}$ \\
Mode II debonding displ. & $\bar{u}_{I I}^{f}=0.2 \mathrm{~mm}$ \\
Shear strength & $\bar{t}_{I I}=40 \mathrm{~N} / \mathrm{mm}$ \\
Mode I Fracture energy & $G_{I}=1 \mathrm{~N} / \mathrm{mm}$ \\
Mode II Fracture energy & $G_{I I}=4 \mathrm{~N} / \mathrm{mm}^{2}$ \\
\hline & Frictional Parameters \\
\hline Normal elastic stiffness & $K_{n}^{f}=50000 \mathrm{~N} / \mathrm{mm}^{3}$ \\
Tangential elastic stiffness & $K_{t}^{f}=5000 \mathrm{~N} / \mathrm{mm}^{3}$ \\
Frictional coefficient & $\alpha=0.8391$ \\
Dilatancy coefficient & $\beta=0$ \\
\hline \hline
\end{tabular}

Table 1: Model constitutive parameters used for the numerical simulations.

theory is given, in terms of imposed displacement $u$ and relevant load $P$, by

$$
\begin{aligned}
& u=4 a^{2} \sqrt{\frac{G_{I}}{3 E h^{3}}} \\
& P=\frac{3 E I}{2 a^{3}} u
\end{aligned}
$$

526 with $I=b h^{3} / 12$. Results of numerical simulation are plotted in Fig.11 in terms

${ }_{527}$ of horizontal normal stress at the initial delamination condition. Results of ${ }_{528}$ analytical solution and numerical simulations are compared in Figure 12. The 529 numerical results properly reproduce the analytical solution in the descending 530 branch, whereas the numerical solution is less stiff than the analytical one in the ${ }_{531}$ initial elastic path. In fact the analytical solution is based on the linear elastic ${ }_{532}$ fracture mechanics theory, which assumes an ideally brittle traction-separation 
law. As a consequence, the initially elastic behaviour of the interface produces a less stiff response.

The initial elastic behaviour assumed for the interface can be considered as a penalty approach in order to impose the rigidity constrain. The interface elastic stiffness, or equivalently the tensile strength $\bar{t}_{I}$ for fixed fracture energy, represents the penalty parameter. Is well known that analytical solution can not be caught by penalty method and, as penalty parameter increases over a specific value, error in numerical solution increases too. Such a problem has been observed in the numerical solution of the DBC test for $\bar{t}_{I}>40 \mathrm{~N} / \mathrm{mm}^{2}$.

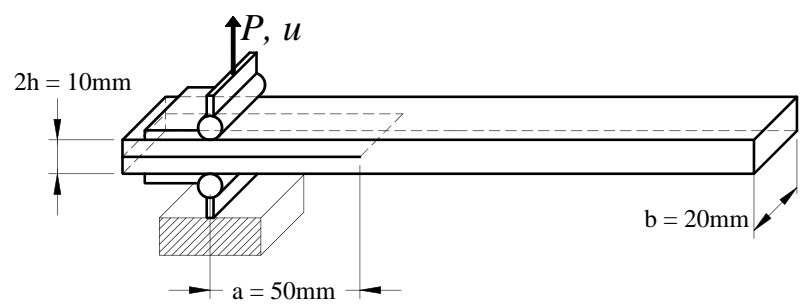

Figure 10: Sizes and geometry of specimen for the double cantilever beam test.

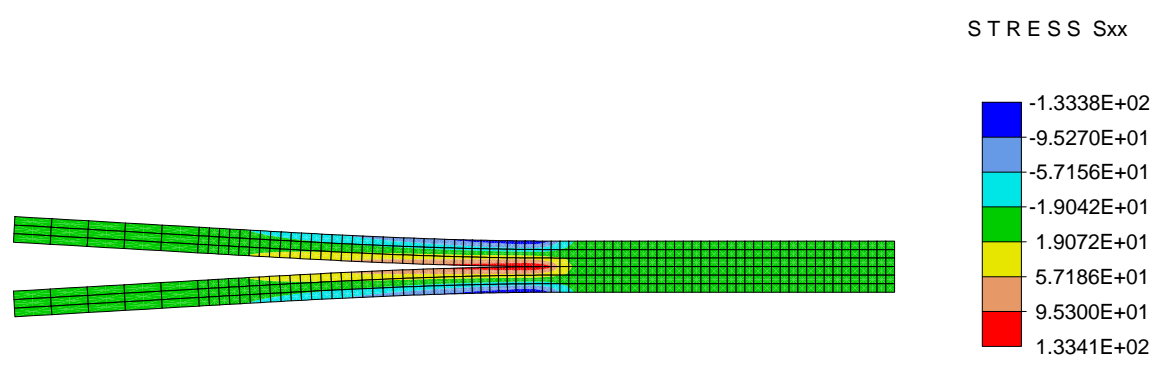

Figure 11: Map of normal stress Sxx obtained by the numerical simulation of the double cantilever beam test at the initial delamination condition. 


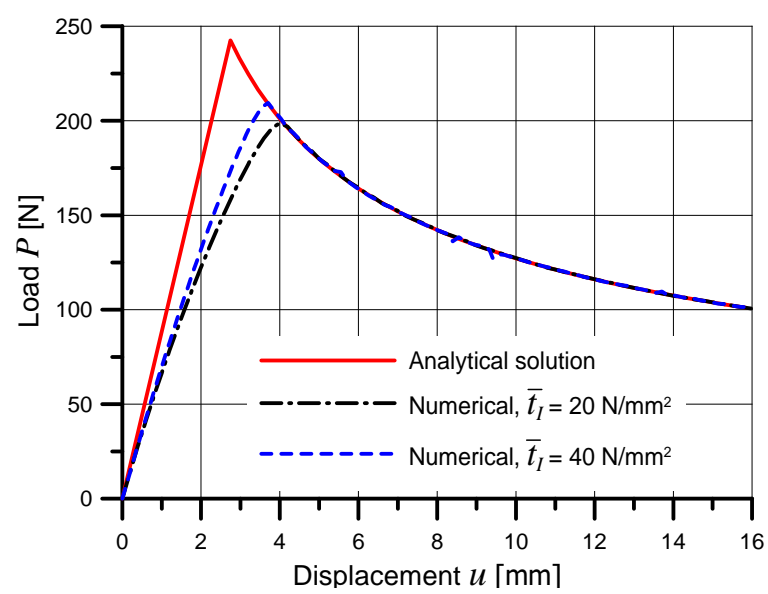

Figure 12: Response of double cantilever beam test, in terms of applied load vs imposed displacement. Analytical solution and numerical solutions with two different tensile strengths.

The analytical solution is defined in terms of crack opening displacement $d$ (see Figure 13) and applied load $P$, and it is derived in the framework of fracture mechanics and beam theory. Analytical solution at the first delamination, for crack length less than the beam half-span $(a \leq L)$, is given in Mi et al. (1998) 552 as

$$
\begin{aligned}
P & =\frac{1}{a} \sqrt{\frac{8 E I b}{\frac{8}{G_{I}}\left(\frac{3 C-L}{4 L}\right)^{2}+\frac{3}{8 G_{I I}}\left(\frac{C+L}{L}\right)^{2}}} \\
d & =\frac{2 P a^{3}}{3 E I} \frac{3 C-L}{4 L}
\end{aligned}
$$

553 with $I=b h^{3} / 12$. The second analytical solution, for crack extended behind the 554 beam mid-span $(a \geq L)$, was initially given in Mi et al. (1998), but a corrected

555 formulation was proposed in Tenchev and Falzon (2007) in term of crack opening displacement

$$
d=\frac{P}{E I} \frac{\left(a^{3}+3 a^{2} L-L^{3}\right)(L+C)-4 a^{3} L}{6 L}
$$

557 and the applied load can be derived by the following mixed mode interaction

558 fracture criterion

$$
\frac{Y_{I}}{G_{I}}+\frac{Y_{I I}}{G_{I I}}=1
$$


where $Y_{I}$ and $Y_{I I}$ are the following energy release rates

$$
\begin{aligned}
Y_{I} & =\frac{P^{2} L^{2}}{8 b E I}\left[\frac{a^{2}}{2 L^{2}}\left(\frac{C}{L}-3\right)^{2}+\frac{a}{2 L}\left(\frac{C}{L}+1\right)\left(5 \frac{C}{L}-13\right)+\left(\frac{C}{L}+1\right)\left(\frac{C}{L}+3\right)\right] \\
Y_{I I} & =\frac{P^{2} L^{2}}{8 b E I}\left[\frac{3 a^{2}}{8 L^{2}}\left(\frac{C}{L}+1\right)^{2}-\frac{a}{L}\left(\frac{C}{L}+1\right)\left(2 \frac{C}{L}+1\right)+\frac{1}{2}\left(\frac{C}{L}+1\right)\left(5 \frac{C}{L}+1\right)\right] .
\end{aligned}
$$
$u=1 \mathrm{~mm}$, is plotted in Fig.14.

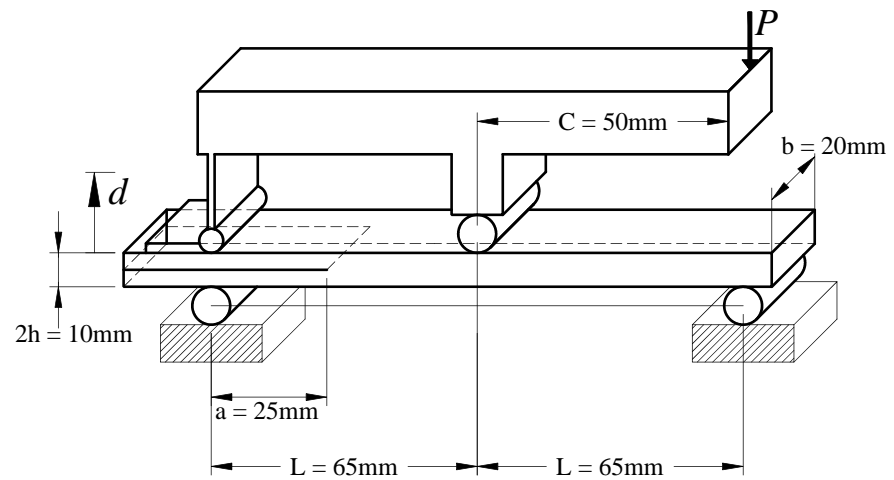

Figure 13: Sizes and geometry of specimen for the mixed mode test (MMBT).

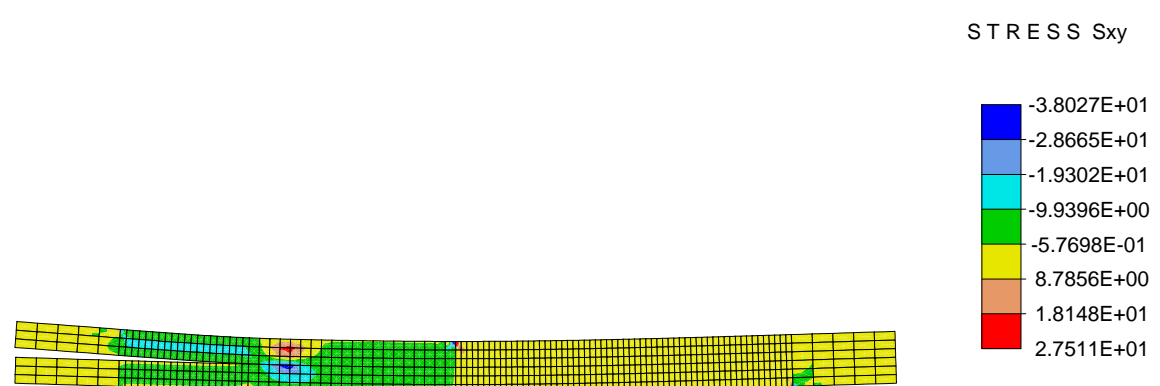

Figure 14: Map of tangential stress Sxy obtained by the numerical simulation of the MMBT at the loading condition of imposed displacement $u=1 \mathrm{~mm}$. 


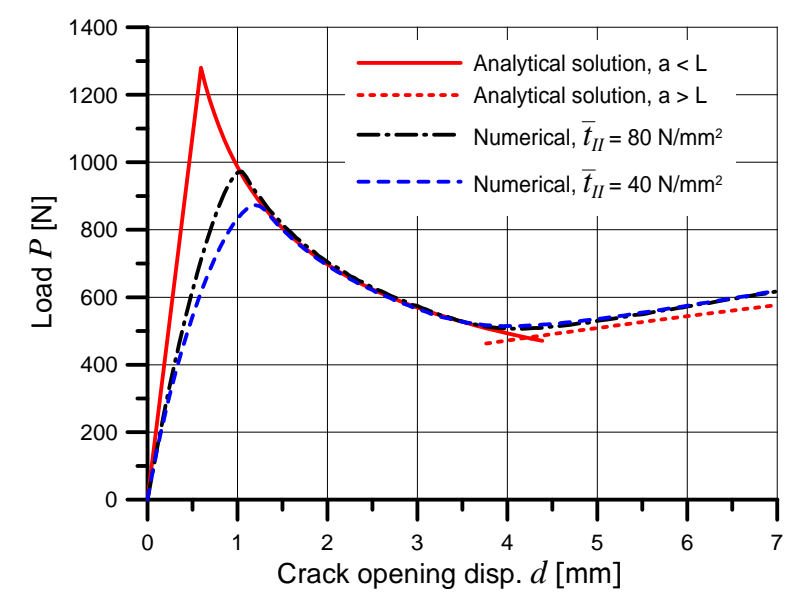

Figure 15: Response of mixed mode bending test, in terms of applied load vs crack opening displacement. Analytical solution and numerical solutions with two different tensile strengths.

\subsection{ENF test}

The third numerical simulation is the four points bend end-notched flexure test (4ENF), represented in Figure 16 with the relevant sizes.

The analytical solution can be developed in the framework of beam theory and fracture mechanics (Martin and Davidson, 1999) and is given by

$$
\begin{aligned}
P & =\frac{4}{3} \frac{B}{L-D} \sqrt{E h^{3} G_{I I}} \\
u & =P \frac{(L-D)^{2}}{24 E I}(3 a+4 D-2 L)
\end{aligned}
$$

571 with $I=b h^{3} / 12$. Map of tangential stress obtained by the numerical simula572 tion, at the loading condition of imposed displacement $u=3 \mathrm{~mm}$, is plotted in 573 Fig.17. Results of numerical simulation and analytical solution are compared ${ }_{\mathbf{5 7 4}}$ in Figure 18 in terms of applied load $P$ and relevant displacement $u$, for both 575 the two set of constitutive parameters. Moreover, two solutions with positive 576 frictional coefficients have been carried out and results are compared to the 577 analytical (frictionless) solution.

578 4ENF test is known in literature (Schuecker and Davidson, 2000) for its 579 accuracy on the determination of mode II delamination toughness, which is 580 influenced by frictional effects, over than by ratio between inner span and outer 


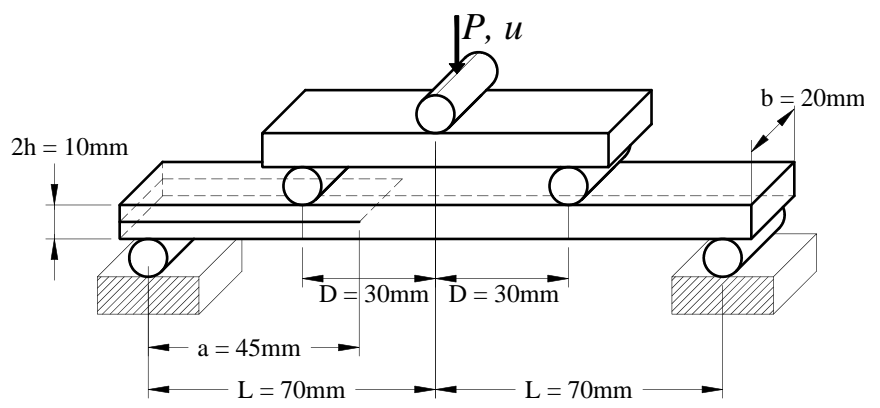

Figure 16: Sizes and geometry of specimen for the four point bend end-notched flexure test (4ENF).

STRESS Sxy
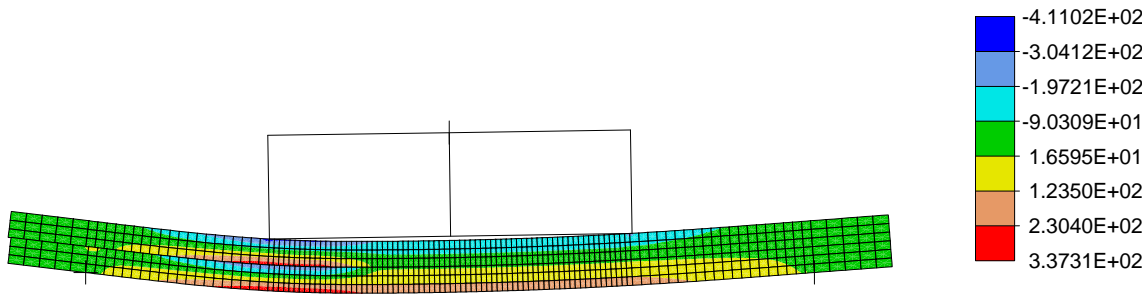

Figure 17: Map of tangential stress Sxy obtained by the numerical simulation of the 4ENF at the loading condition of imposed displacement $u=3 \mathrm{~mm}$.

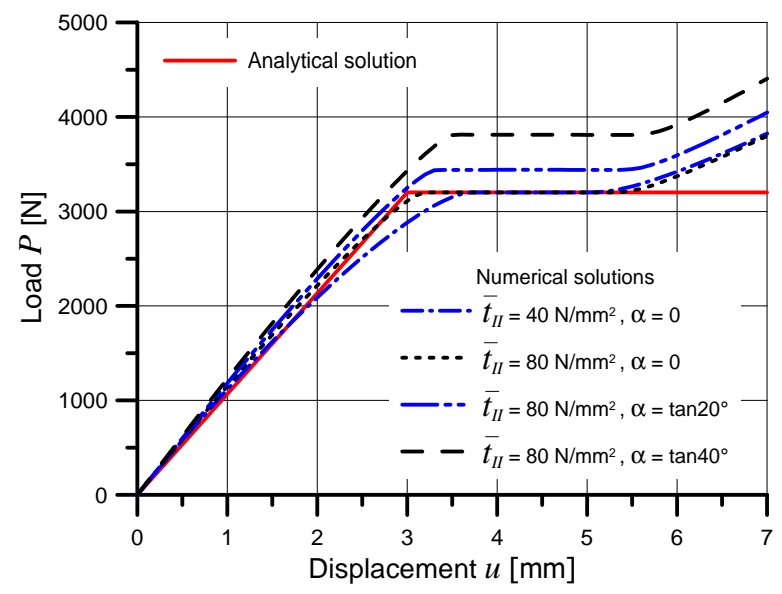

Figure 18: Response of $4 \mathrm{ENF}$ test, in terms of applied load vs imposed displacement. Analytical solution and numerical solutions with two different tensile strengths and with three different frictional coefficients. 
span ( $D / L$ with reference to Fig.16).

The proposed model can numerically reproduce the standard $4 \mathrm{PBT}$ and it can also take into account the presence of frictional effects. Numerical simulations with two frictional coefficients $\left(\alpha=\tan 20^{\circ}\right.$ and $\left.\alpha=\tan 40^{\circ}\right)$ and null dilatancy coefficient $(\beta=0)$ have been performed and results are shown in Fig.18 and compared the frictionless numerical results and with analytical solution. Diagrams plotted if Fig. 18 show that applied load at delamination condition is $7.5 \%$ greater than frictionless response, for the frictional coefficient $\alpha=\tan 20^{\circ}$, and $19 \%$ greater than frictionless response, for the frictional coefficient $\alpha=\tan 40^{\circ}$.

Finally, the differences between numerical and analytical responses in the initial elastic branch, observable in the three delamination tests, are intrinsic to the cohesive zone formulations. In fact, the analytical solution are developed in the linear fracture mechanics, for which the behaviour is linear elastic up to delamination starts. On the contrary, in cohesive zone models the delamination phenomenon is subsequent the nonlinear behaviour in the cohesive zone and the response is less stiff than the analytical one.

\section{Closing remarks}

The paper presents as a main innovative finding an interface unified constitutive framework based on a single damage variable in a thermodynamic consistent context, which has a proper free energy, dissipation function, activation function and evolution rules, all derived in the context of dissipative mechanics with internal variables.

The proposed CZM, produces two independent fracture energies, $G_{I}$ in pure mode I debonding condition and $G_{I I}$ in pure mode II debonding one. $G_{I}$ and $G_{I I}$, as analytically shown, are minimum and maximum values of the work-ofseparation for any proportional and non-proportional loading paths. The model can also evaluates the presence of frictional tractions both at the fully debonded zones and at the partially debonded ones. 
The proposed model is able to accurately reproduce with a unique set of few constitutive parameters, very different and general proportional and non${ }_{612}$ proportional, monotonic and cyclic, loading paths, either in opening mode or in

613 sliding mode and in any mixed condition, recovering also closing conditions and ${ }_{614}$ frictional effects.

Finally, three classical delamination tests (DBC, MMB, 4ENF) have been 616 numerically reproduced and the results compared with the analytical ones, show-

${ }_{617}$ ing good agreement.

\section{Acknowledgment}

A grant from the Italian Ministry for University and Research (MIUR) and University of Palermo for FFR 2012-2014, 2014-ATE-0243 project "Procedure multiscala per l'analisi di strutturale: aspetti teorici, meccanici e numerici" is acknowledged. The authors also acknowledge the financial support from Project "SLIM", linea intervento 4.1.1.1 del P.O. FERS Sicilia 2013

\section{References}

Alfano, G., Crisfield, M., 2001. Finite element interface models for the delamination analysis of laminated composites: Mechanical and computational issues. Int. J. Num. Meth. Eng. 50 (7), 1701-1736.

Alfano, G., Sacco, E., 2006. Combining interface damage and friction in a cohesive-zone model. Int. J. Num. Meth. Eng. 68 (5), 542-582.

Allix, O., LadevÃl'ze, P., Corigliano, A., 1995. Damage analysis of interlaminar fracture specimens. Comp. Struct. 31 (1), 61-74.

ASTM, 2006. Standard test method for mixed mode i-mode ii interlaminar fracture toughness of unidirectional fiber reinforced polymer matrix composites: Technical report astm d6671/d6671m. Tech. rep., ASTM International.

Barenblatt, G., 1962. The mathematical theory of equilibrium cracks in brittle fracture. Advances in Applied Mechanics 7, 55-129. 
Benzeggagh, M. L., Kenane, M., 1996. Measurement of mixed-mode delamination fracture toughness of unidirectional glass/epoxy composites with mixedmode bending apparatus. Compos. Sci. Technol. 56, 439-449.

Benzerga, D., Haddi, A., Seddak, A., Lavie, A., 2008. A mixed-mode damage model for delamination growth applied to a new woven composite. Comp. Mat. Sci. 41 (4), 515-521.

Camanho, P., DÃąvila, C., De Moura, M., 2003. Numerical simulation of mixedmode progressive delamination in composite materials. J. Comp. Materials 37 (16), 1415-1438.

Coleman, B., Noll, W., 1963. The thermodynamics of elastic materials with heat conduction and viscosity. Arch. Rat. Mech. Analysis 13, 167-178.

Corigliano, A., 1993. Formulation, identification and use of interface models in the numerical analysis of composite delamination. Int. J. Solids Structures 30 (20), 2779-2811.

Corigliano, A., Allix, O., 2000. Some aspects of interlaminar degradation in composites. Comp. Meth. Appl. Mech. Eng. 185 (2-4), 203-224.

Corigliano, A., Ricci, M., 2001. Rate-dependent interface models: Formulation and numerical applications. Int. J. Solids Structures 38 (4), 547-576.

Crews, J., Reeder, J., 1998. A mixed-mode bending apparatus for delamination testing, technical memorandum 100662. Tech. rep., NASA Lagley.

Daudeville, L., Allix, O., Ladevéze, P., 1995. Delamination analysis by damage mechanics: Some applications. Comp. Engng 5 (1), 17-24.

Dimitri, R., Trullo, M., Zavarise, G., De Lorenzis, L., 2014. A consistency assessment of coupled cohesive zone models for mixed-mode debonding problems. Frattura ed Integrita Strutturale 8 (29), 266-283.

Dugdale, D., 1960. Yielding of steel sheets containing slits. J. Mech. Phys. Solids. 8, 100-104. 
Ganghoffer, J., Schultz, J., 1997. Interactions between adhesion and frictionâĂ ̌̌i. theoretical aspects. J. Mech. Phy. Solids 45 (1), 151-174.

Giambanco, G., Fileccia Scimemi, G., 2006. Mixed mode failure analysis of bonded joints with rate-dependent interface models. Int. J. Num. Meth. Engng. 67 (8), 1160-1192.

Guiamatsia, I., Nguyen, G., 2014. A thermodynamics-based cohesive model for interface debonding and friction. Int. J. Solids Structures 51 (3-4), 647-659.

Högberg, J., 2006. Mixed mode cohesive law. Int. J. Fracture 141 (3-4), 549-559.

Kolluri, M., Hoefnagels, J. P. M., van Dommelen, J. A. W., Geers, M. G. D., 2014. Irreversible mixed mode interface delamination using a combined damage-plasticity cohesive zone enabling unloading. Int. J. Fracture $185,77-95$.

Martin, R., Davidson, B., 1999. Mode ii fracture toughness evaluation using four point bend, end notched flexure test. Plas. Rubber Compos. Proc. Appl. 28 (8), 401-406.

McGarry, J., ÂŞ MÃąirt Ãyn, ., Parry, G., Beltz, G., 2014. Potential-based and non-potential-based cohesive zone formulations under mixed-mode separation and over-closure. part i: Theoretical analysis. J. Mech. Physics Solids 63 (1), $336-362$.

Mi, Y., Crisfield, M., Davies, G., Hellweg, H.-B., 1998. Progressive delamination using interface elements. J. Compos. Materials 32 (14), 1246-1272.

Mosler, J., Scheider, I., 2011. A thermodynamically and variationally consistent class of damage-type cohesive models. J. Mech. Physics Solids 59 (8), 16471668.

Musto, M., Alfano, G., 2013. A novel rate-dependent cohesive-zone model combining damage and visco-elasticity. Computers and Structures 118, 126-133. 
Park, K., Paulino, G., Roesler, J., 2009. A unified potential-based cohesive model of mixed-mode fracture. J. Mech. Physics Solids 57 (6), 891-908.

Parrinello, F., Failla, B., Borino, G., 2009. Cohesive-frictional interface constitutive model. Int. J. Solids Structures 46 (13), 2680-2692.

Parrinello, F., Marannano, G., Borino, G., Pasta, A., 2013. Frictional effect in mode ii delamination: Experimental test and numerical simulation. Eng. Fract. Mech. 110, 258-269.

Reeder, J., Crews, J., 1990. Mixed-mode bending method for delamination testing. AIAA Journal 28, 1270-1276.

Sacco, E., Lebon, F., 2012. A damage-friction interface model derived from micromechanical approach. Int. J. Solids Structures 49 (26), 3666-3680.

Schuecker, C., Davidson, B., 2000. Evaluation of the accuracy of the four-point bend end-notched flexure test for mode ii delamination toughness determination. Compos. Sci. Tech. 60 (11), 2137-2146.

Serpieri, R., Alfano, G., 2011. Bond-slip analysis via a thermodynamically consistent interface model combining interlocking, damage and friction. Int. J. Num. Meth. Engng. 85 (2), 164-186.

Serpieri, R., Sacco, E., Alfano, G., 2015. A thermodynamically consistent derivation of a frictional-damage cohesive-zone model with different mode $\mathrm{i}$ and mode ii fracture energies. Eur. J. Mech., A/Solids 49, 13-25.

Spada, A., Giambanco, G., Rizzo, P., 2009. Damage and plasticity at the interfaces in composite materials and structures. Comp. Meth. Appl. Mech. Eng. 198 (49-52), 3884-3901.

Tenchev, R., Falzon, B., 2007. A correction to the analytical solution of the mixed-mode bending (mmb) problem. Compos. Sci. Tech. 67 (3-4), 662-668. 

van den Bosch, M., Schreurs, P., Geers, M., 2006. An improved description of the exponential xu and needleman cohesive zone law for mixed-mode decohesion.

$717 \quad$ Eng. Fract. Mech. 73 (9), 1220-1234.

${ }_{118}$ Xu, X. P., Needleman, A., 1993. Void nucleation by inclusion debonding in a crystal matrix. Modell. Simulation Mater. Sci. Eng. 2, 417-418.

Zienkiewicz, O., Taylor, 2000. The Finite Element Method. 5th Edition. Butterworth-Heinemann Press.

${ }_{722}$ Zreid, I., Fleischhauer, R., Kaliske, M., 2013. A thermomechanically coupled ${ }_{723}$ viscoelastic cohesive zone model at large deformation. Int. J. Solids Structures $724 \quad 50,4279-4291$. 